01,11

\title{
Стабилизация роста перлитной колонии в результате взаимодействия углерода с дилатациями решетки
}

\author{
(C) И.К. Разумов
}

Институт физики металлов УрО РАН, Екатеринбург, Россия

E-mail: rik@imp.uran.ru

(Поступила в Редакцию 4 апреля 2017 г.)

Предложенная ранее модель перлитного превращения развивается с учетом возможного взаимодействия углерода с дилатациями решетки, возникающими в аустените вблизи перлитной колонии. Создаваемые колонией нормальные напряжения стимулируют автокатализ пластин, а тангенциальные напряжения способствуют стабилизации фронта превращения. Обсуждается механизм ветвления феррита, который может играть существенную роль в кинетике перлитного и бейнитного превращений.

Работа выполнена в рамках государственного задания ФАНО России (тема „Магнит“, № 01201463328).

DOI: 10.21883/FTT.2017.10.44954.110

\section{1. Введение}

Перлитное превращение в углеродистой стали [1-3] относится к классу эвтектоидных превращений, при которых исходная метастабильная фаза претерпевает распад ниже эвтектоидной температуры $T_{\text {eutec }}$ на две новые фазы по сценарию роста колоний. В случае перлитного превращения в стали при $T_{\text {eutec }}<1000 \mathrm{~K}$ метастабильный аустенит ( $\gamma$-Fe, ГЦК решетка) распадается на феррит с низкой растворимостью углерода $(\alpha-\mathrm{Fe}$, ОЦК решетка) и цементит, содержащий 25 at.\% углерода ( $\theta$-фаза, орторомбическая решетка); в результате обычно формируется регулярная пластинчатая структура. Эвтектоидные превращения наблюдаются также в сплавах $\mathrm{Zn}-\mathrm{Al}[4], \mathrm{Cu}-\mathrm{Al}[5], \mathrm{Au}-\mathrm{In}[6]$ и др.

Несмотря на сходство перлита со структурами, возникающими при спинодальном распаде (SD) [7], классический SD невозможен в $\gamma$-Fe, поскольку энергия смешения углерода положительна в этой фазе [8]. Теоретические исследования фокусировались на исследовании стационарного роста перлитной колонии с плоским фронтом [9-14], что позволило, в частности, установить температурную зависимость межпластиночного расстояния $\lambda \approx 1 /\left(T-T_{\text {eutec }}\right)$, в согласии с экспериментом. В работе [11] впервые была сформулирована гипотеза, недавно подтвержденная phase-field моделированием [15], что устойчивый рост перлитной колонии обеспечивается ускорением диффузии углерода на ее фронте. В работе [15] было показано, что в отсутствие этого ускорения ферритные пластины быстро прорастают в аустенит, после чего регулярная структура колонии разрушается. В то же время, в работе [16] цитируется мнение Хиллерта, согласно которому трудно ожидать значительного ускорения диффузии на фронте превращения, учитывая и без того быструю диффузию в объеме фаз.

Зарождение перлитной колонии обсуждалось качественно в работе [17], где было отмечено, что между линиями фазовой диаграммы $A_{3}$ и $A_{c m}$ (рис. 1) экстраполированными в область температур $T<T_{\text {eutec }}$, аустенит пересыщен по отношению как к ферриту, так и к цементиту. Углерод вытесняется из феррита, способствуя зарождению цементита на границе с ферритом. В свою очередь, рост выделения цементита приводит к обеднению углеродом прилегающей области, способствуя зарождению феррита. Процесс может описываться в терминах флуктуационного зарождения, причем вероятность зарождения каждой из фаз зависит от локального состава.

В работе [19] был впервые рассмотрен автокаталитический сценарий распада метастабильных фаз, и обсуж-

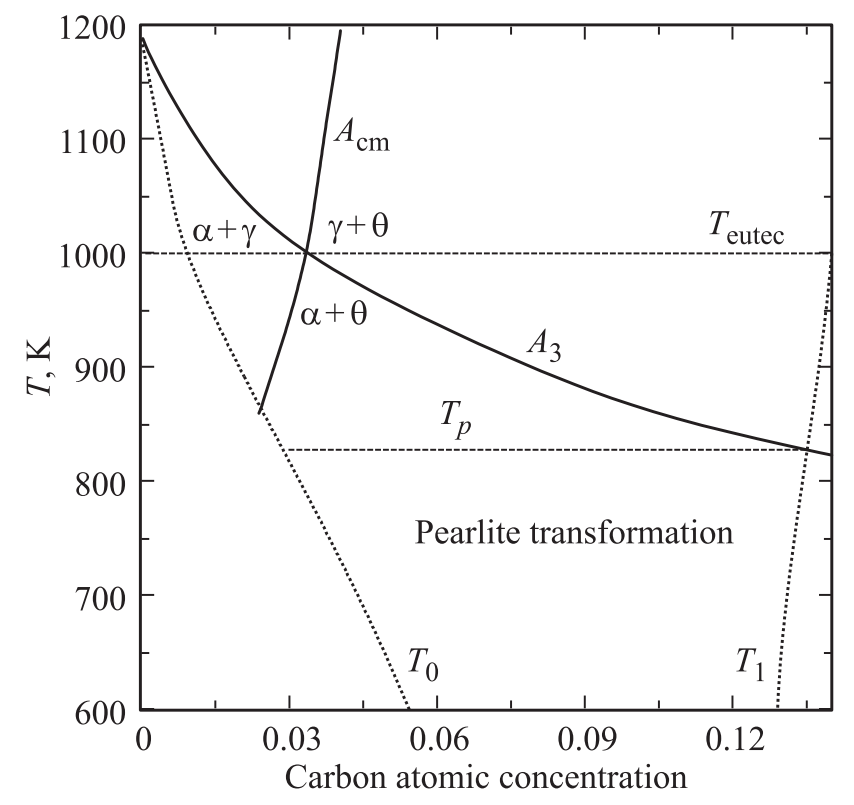

Рис. 1. Диаграмма превращений в системе $\mathrm{Fe}-\mathrm{C}$, построенная в рамках модели [18] с уточненной параметризацией (см. приложение). $A_{3} A_{c m}$ - границы двухфазных областей $\alpha+\gamma$, $\gamma+\theta$ соответственно; $T_{0}, T_{1}-$ кривые потери устойчивости аустенита относительно превращений $\gamma \rightarrow \alpha, \gamma \rightarrow \theta$. 


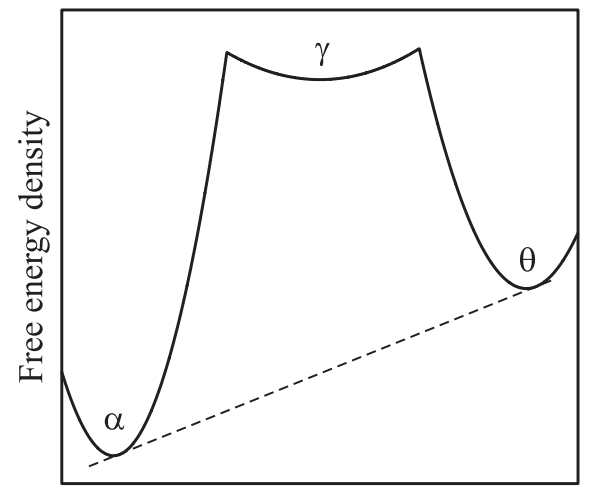

Carbon atomic concentration

Pис. 2. Свободные энергии фаз в перлитной области диаграммы превращений (схематичное изображение). Отсутствуют условия равновесия $\alpha / \gamma$ и $\gamma / \theta$ при сохранении основного равновесия $\alpha / \theta$, что приводит к автокаталитическому распаду $\gamma$-фазы.

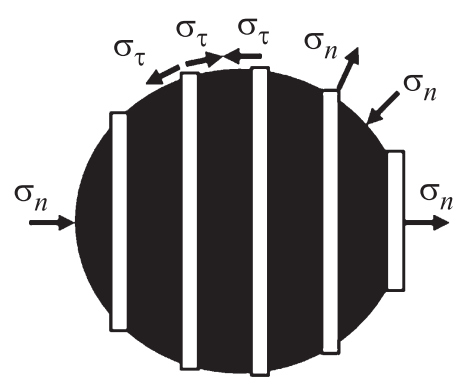

Рис. 3. Нормальные $\left(\sigma_{n}\right)$ и тангенциальные $\left(\sigma_{\tau}\right)$ напряжения в окрестности перлитной колонии (схематичное изображение).

далась возможность реализации подобного механизма при перлитном превращении. Данный механизм отличается как от классического спинодального распада, так и от флуктуационного зарождения. Его специфика состоит в том, что при специальном соотношении энергий фаз (рис. 2) исходная фаза может быть стабильной относительно малых флуктуаций состава, но локально теряет устойчивость при контакте с продуктами превращения, т. е. в тех участках, где в силу стремления системы к локальному равновесию достигается изменение концентрации выше критического. В результате один из продуктов превращения стимулирует быстрое зарождение другого, и распад развивается по сценарию роста колоний. Позднее в работе [15] рассматривалось автокаталитическое зарождение выделений на границе зерна в модели, качественно имитирующей перлитное превращение. Подробное обсуждение автокаталитического механизма распада метастабильных фаз приводится в обзоре, посвященном теории фазовых превращений в стали [20].

В работе [18] предложена модель перлитного превращения с первопринципной параметризацией. Автокаталитический механизм распада в этой модели оказался возможным благодаря гипотезе, что зарождение цементита на границе с ферритом облегчается (линия потери устойчивости $T_{1}$ смещается влево, рис. 1) в резуль- тате формирования особой промежуточной структуры (Metastable Intermediate Structure, MIS) на межфазной границе аустенит/феррит, в соответствии с недавними $a b$ initio расчетами [21]. В отличие от работы [15] в модели [18] не использовались предположения о плоском фронте превращения и об ускорении диффузии на фронте, а автокатализ был разрешен по всему объему материала. В результате наблюдались морфологии распада, качественно подобные перлитным (согласно экспериментам, фронт колонии не обязательно является плоским [2,22]). Именно отсутствие плоского фронта способствовало в этих расчетах развитию автокатализа и формированию пластинчатой структуры. В то же время в экспериментах ламели обычно ориентированы по нормали к фронту превращения, что не вполне согласуется с этими расчетами.

При перлитном превращении когерентность решетки на фронте колонии теряется полностью или частично [23-25]. Поэтому в большинстве теорий принимается, что упругие напряжения в аустените не играют существенной роли в кинетике превращения. Однако такой взгляд на роль напряжений является слишком упрощенным. Даже при отсутствии кооперативного смещения атомов в системе неизбежно возникают решеточные дилатации, обусловленные диффузией углерода и различием объемов фаз. Концентрационные напряжения, обусловленные изменением локальной концентрации углерода вблизи фронта колонии, были учтены и привели к заметному ускорению превращения [26]. Кроме концентрационных напряжений вблизи колонии действуют напряжения, обусловленные присутствием феррита и цементита, несводимые к локальной концентрации углерода.

Как видно из рис. 3, в результате различия объемов аустенита, феррита и цементита вблизи торцов колонии возникают нормальные, а в области фронта - тангенциальные напряжения. Поэтому на торцах колонии решетка аустенита растягивается вблизи феррита и сжимается в окрестности цементита. Напротив, перед фронтом колонии решетка аустенита может растягиваться вблизи цементита и сжиматься в окрестности феррита. Поскольку атомы углерода являются центрами дилатации, они диффундируют из областей сжатия в области растяжения. Учет этого обстоятельства способствует формированию регулярной структуры и повышает температуру старта автокатализа, при этом ламели ориентируются по нормали к фронту колонии в согласии с экспериментом.

\section{2. Формулировка модели}

Запишем функционал свободной энергии ГинзбургаЛандау в виде

$$
F=\int\left(f_{e f f}(c, T)+\frac{k_{c}}{2}(\nabla c)^{2}\right) d \mathbf{r}+F_{e l . i n},
$$

где $F_{\text {el.in }}$ - энергия деформационного взаимодействия углерода с напряжениями, создаваемыми растущей колонией; $f_{\text {eff }}(c, T)=\min \left\{f_{\alpha}(c, T), f_{\gamma}(c, T), f_{\theta}(c, T)\right\}-$ 
эффективная плотность свободной энергии ненапряженного состояния, зависящая от локальной концентрации углерода и температуры; $f_{\gamma(\alpha, \theta)}(c, T)$ - локальные плотности свободной энергии аустенита (феррита, цементита), вычисляемые аналогично модели [18,20,27]; $k_{c}$ - параметр, определяющий ширину межфазной границы. Определение $f_{e f f}(c, T)$ предполагает быструю перестройку решеток, так что в заданной точке пространства всегда наблюдается фаза с наименьшей свободной энергией.

Опишем основные положения используемого подхода $[18,20,27]$. Энергии $\alpha$ и $\gamma$ фаз в присутствии углерода (или без него) были рассчитаны $a b$ initio для парамагнитного и ферромагнитного состояний. Энергии этих фаз на промежуточных температурах получены в согласии с моделью Огучи для температурной зависимости спинового коррелятора [28]. Магнитная энтропия учитывается с привлечением теоремы Гельмана-Фейнмана [29]. Конфигурационная энтропия углерода учитывает, что атомы углерода в $\alpha$-фазе распределяются по трем подрешеткам октаэдрических междоузлий, а в $\gamma$-фазе могут занимать только четвертую часть октаэдрических междоузлий [30]. Температурная зависимость свободной энергии стехиометрического цементита берется из CALPHAD [31] или $a b$ initio pacчетов [32]. Концентрационная зависимость энергии цементита выбирается в эффективном виде [18], обеспечивая реализацию перехода $\gamma \rightarrow \theta$ (условие потери устойчивости) при концентрациях $c=0.12-0.15$, т.е. вдали от стехиометрического состава цементита. Физическим основанием для такого выбора являются вероятное появление промежуточной структуры (MIS) на границе аустенит/феррит, облегчающей переход в соответствии с ab initio расчетами [21], а также предпочтительность зарождения цементита на границе с ферритом, обусловленная упругими напряжениями. Параметризация модели незначительно уточняется (ср. с [18] с привлечением экспериментальных данных [30] для энергий растворения углерода (приложение).

При охлаждении возрастает ближний магнитный порядок в $\alpha$-фазе, что приводит к понижению энергии этой фазы. Кроме того, энергия цементита при охлаждении понижается быстрее, чем энергия аустенита. В результате реализуется переход от стабильных равновесий $\gamma / \alpha$ и $\gamma / \theta$ сначала к метастабильным равновесиям, а затем к их отсутствию при сохранении основного равновесия $\alpha / \theta$. Для качественных выводов в настоящей работе существенно только, что ниже критической температуры $\left(T<T_{p}\right)$ функция $f_{e f f}(c)$ представляет собой трехъямный потенциал, для которого (рис. 2) отсутствуют метастабильные равновесия между аустенитом и продуктами распада, как и ранее [18].

Плотность упругой энергии взаимодействия углерода с напряжениями определяется формулой:

$$
f_{e l . i n}(\mathbf{r})=\frac{1}{2} \sigma_{i j}(\mathbf{r}) \varepsilon_{i j}^{(C)} c(\mathbf{r}),
$$

где $\sigma_{i j}(\mathbf{r})-$ упругие напряжения, создаваемые растущей колонией в точке $\mathbf{r}, \varepsilon_{i j}^{(C)}-$ деформации, создаваемые одиночным атомом углерода. Если атом углерода создает только дилатации:

$$
\varepsilon_{i j}^{(C)}=\frac{\sqrt{2}}{2} \delta_{i j} v^{(C)},
$$

выражение (2) принимает вид:

$$
f_{\text {el.in }}(\mathbf{r})=\frac{\sqrt{2}}{4} v^{(C)} \sigma_{i i}(\mathbf{r}) c(\mathbf{r}) .
$$

Напряжения определяются функционалом от деформаций решетки в окрестности точки $\mathbf{r}$

$$
\sigma_{i i}(\mathbf{r})=\frac{1}{L^{2}} \int K\left(\left|\mathbf{r}-\mathbf{r}^{\prime}\right|\right) q\left[c\left(\mathbf{r}^{\prime}\right)\right] d \mathbf{r}^{\prime},
$$

где $L$ - размер образца, $q\left[c\left(\mathbf{r}^{\prime}\right)\right]-$ функция, отражающая присутствие перлитной колонии в точке $\mathbf{r}^{\prime}$ и определяемая собственными деформациями превращения $e^{\alpha}, e^{\theta}$

$$
q\left[c\left(\mathbf{r}^{\prime}\right)\right]=e^{\theta} \chi_{\theta}\left(\mathbf{r}^{\prime}\right)+e^{\alpha} \chi_{\alpha}\left(\mathbf{r}^{\prime}\right),
$$

где $\chi_{\alpha}\left(\mathbf{r}^{\prime}\right), \chi_{\theta}\left(\mathbf{r}^{\prime}\right)$ - функции формы выделений феррита и цементита, которые определим посредством сглаженных функций Хэвисайда (Heaviside's function): $\chi_{\alpha}\left(\mathbf{r}^{\prime}\right)=h\left[c\left(T_{0}\right)-c\left(\mathbf{r}^{\prime}\right)\right], \chi_{\theta}\left(\mathbf{r}^{\prime}\right)=h\left[c\left(\mathbf{r}^{\prime}\right)-c\left(T_{1}\right)\right]$.

Собственные деформации $e^{\alpha}, e^{\theta}$ будем рассматривать как скаляры, связанные с изменением состава:

$$
e^{\alpha(\theta)}=\kappa\left(c_{a(\theta)}^{e}-c_{0}\right),
$$

где $\kappa \equiv v^{(C)}$ - концентрационный коэффициент объемного расширения решетки, $c_{0}-$ исходная средняя концентрация углерода, $c_{\alpha(\theta)}^{e}-$ равновесные концентрации углерода в феррите и цементите, $c_{\alpha}^{e} \approx 0, c_{\theta}^{e}=0.25$.

Функция $K\left(\left|\mathbf{r}-\mathbf{r}^{\prime}\right|\right)$ близка по смыслу к функции Грина, известной в теории упругости [33], и принимается в виде $K(r)=K_{0}\left[1+(r / d)^{4}\right]^{-1}$ (далее в расчетах $d / L=0.05)$. Нормальные напряжения растягивают решетку $\gamma$-фазы вблизи феррита и сжимают ее вблизи цементита, поэтому в этом случае $K_{0}>0$. Напротив, выбор $K_{0}<0$ соответствует тангенциальным напряжениям. Рассматриваемая простая модель не позволяет учесть эти напряжения совместно. Однако учитывая, что на торцах колонии преобладают нормальные напряжения, а вблизи фронта колонии могут играть роль тангенциальные напряжения, их влияние на рост колонии можно исследовать независимо.

Напряжения в объеме колонии исключим из рассмотрения, так как они несущественно сказываются на диффузии углерода, которая в объеме колонии мала, так как $D_{\theta} / D_{\gamma} \ll 1$ и $c_{\alpha}^{e} \ll 1$. Учтем, однако, что знак напряжений изменяется на противоположный при переходе через границу колонии, поэтому при определении вклада в функционал свободной энергии $F_{\text {el.in }}$ введем функцию $w(c)$, которая обращается в ноль в $\alpha$ и $\theta$ фазах:

$$
w(c)=h\left[c-c\left(T_{0}\right)\right] h\left[c\left(T_{1}\right)-c\right],
$$

где $h(x)$ - сглаженная функция Хэвисайда. Тогда $F_{\text {el.in }}$ принимает вид, подобный функционалу Кавасаки 


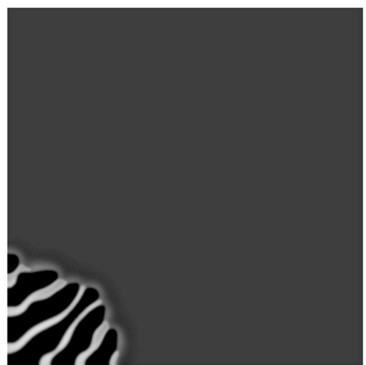

$t=0.24$

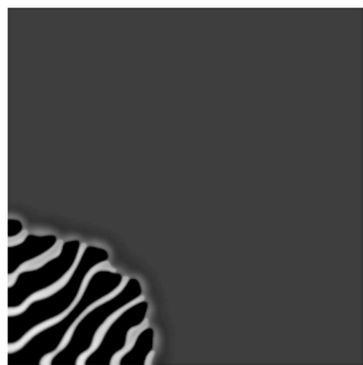

0.33

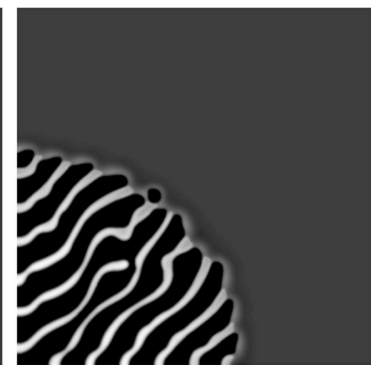

0.51

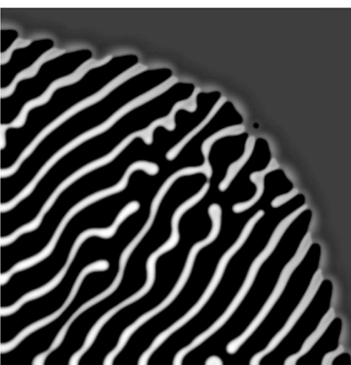

0.78

Рис. 4. Кинетика перлитного превращения при учете нормальных напряжений; $T=900 \mathrm{~K}, c_{0}=0.06,(d / L)^{2} K_{0}=1.9 \mathrm{eV} / \mathrm{at}$.

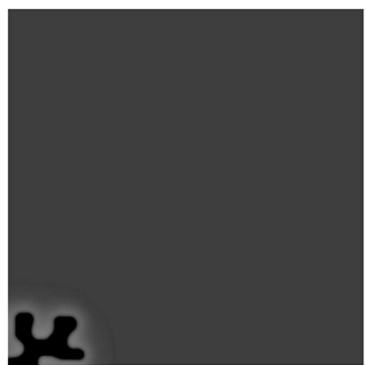

$t=0.74$

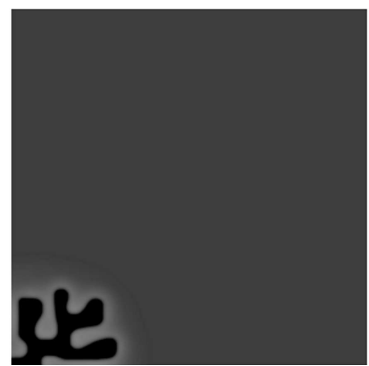

1.1

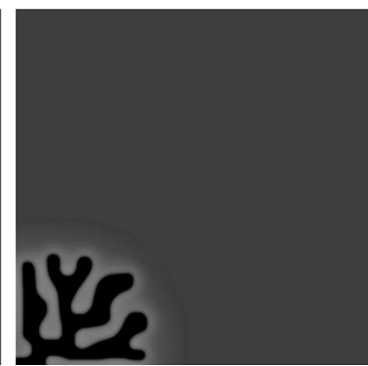

1.57

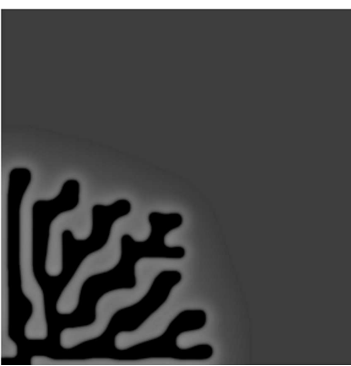

2.75

Рис. 5. Ветвление феррита при учете нормальных напряжений; $T=900 \mathrm{~K}, c_{0}=0.06,(d / L)^{2} K_{0}=0.5 \mathrm{eV} /$ at.

(Kawasaki), который ранее использовали для описания паттернов в различных системах [34]:

$$
F_{e l . i n}=\iint c(\mathbf{r}) w[c(\mathbf{r})] K\left(\left|\mathbf{r}-\mathbf{r}^{\prime}\right|\right) q[c(\mathbf{r})] d \mathbf{r}^{\prime} d \mathbf{r}
$$

Для описания эволюции системы используем стандартное уравнение диффузии [35]:

$$
\frac{\partial c}{\partial t}=-\nabla \mathbf{I}, \quad \mathbf{I}=-\frac{D(c)}{k T} c(1-c) \nabla\left(\frac{\delta F}{\delta c}\right) .
$$

Коэффициент диффузии $D(c)$ выбирается зависящим от концентраций [18] и принимает значения $D_{\alpha(\gamma, \theta)}$ в объеме соответствующих фаз.

\section{3. Результаты моделирования и обсуждение}

Моделирование проводили на квадратной сетке $800 \times 800$ с зеркально-симметричными граничными условиями [36] методом Рунге-Кутта. Время $t$ приводится в безразмерных единицах $L^{2} / D_{\alpha}$, где $L-$ размер стороны квадрата. Различные уровни концентрации углерода обозначаются градациями серого цвета (черный цвет соответствует ферриту, белый - цементиту).

На рис. 4, 5 представлен расчет кинетики превращения при $T=900 \mathrm{~K}$ и разных нормальных напряжениях $\sigma_{n}$, но при отсутствии тангенциальных напряжений $\left(\sigma_{\tau}=0\right)$. Начальное состояние принимали однородным с одиночным зародышем феррита в углу квадрата. Если величина $\sigma_{n}$ выше критической, наблюдается зарождение и рост перлитной колонии (рис. 4), более регулярной, чем в работе [18], причем ламели ориентируются под малым углом к ее фронту. Температура $900 \mathrm{~K}$ выше температуры старта автокатализа в отсутствие напряжений $T_{p}$ (рис. 1). Таким образом, взаимодействие углерода с решеточными дилатациями смещает вверх температуру старта автокатализа, способствуя зарождению перлита.

При понижении $\sigma_{n}$ при той же температуре зарождение цементита не происходит, и наблюдается рост ветвящейся ферритной структуры (рис. 5). Хотя ветвление феррита не наблюдается экспериментально, замеченная тенденция может иметь отношение к перлитному и бейнитному превращениям. В настоящее время в литературе преобладает точка зрения, что пластины перлита размножаются путем боковой репликации [1,2,37], что соответствует автокаталитическому сценарию в нашей модели. Однако в экспериментах Хиллерта наблюдалось размножение пластин путем ветвления [24]. Также наблюдался опережающий рост пластин одного из сортов [38]; а в работе [16] опережающий рост цементитной пластины получен численно при учете напряжений. Более того, обсуждая морфологию ферритной составляющей бейнита, Бхадешиа утверждает, что ферритные планки не являются изолированными, а соединены перемычками в третьем измерении [39]. Механизм размножения ферритных планок бейнита остается на сегодняшний день неясным. Считается, что диффузия углерода может играть определенную роль в их зарождении, но при этом появление цементита является вторичным процессом. 


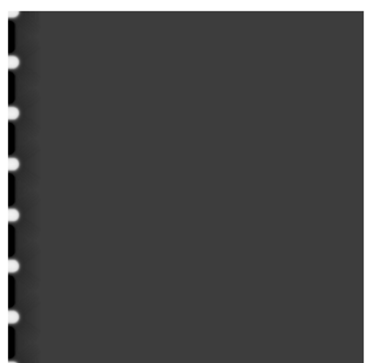

$t=0.05$

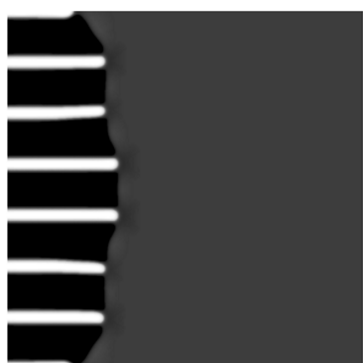

2.5

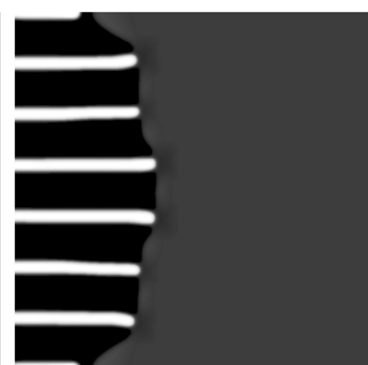

3.4

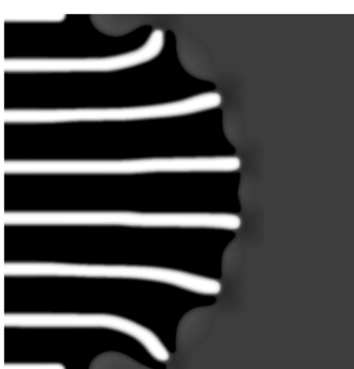

5.5

Рис. 6. Кинетика роста перлитной колонии при учете тангенциальных напряжений; $T=900 \mathrm{~K}, c_{0}=0.06,(d / L)^{2}=-0.25 \mathrm{eV} / \mathrm{at}$.

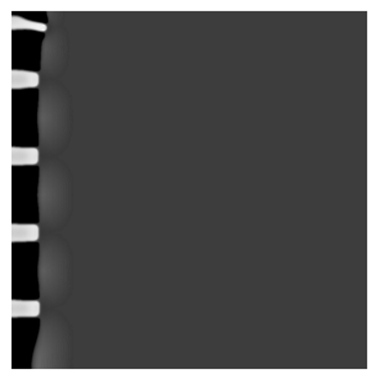

$t=0.08$

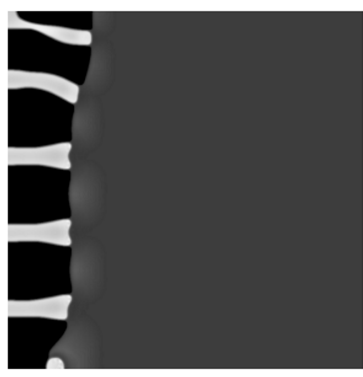

0.20

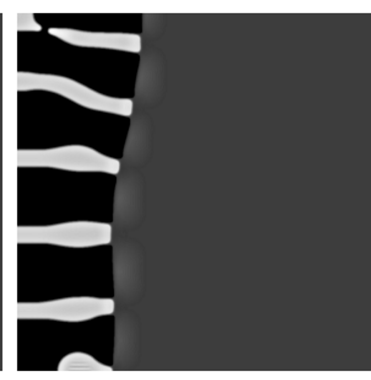

0.45

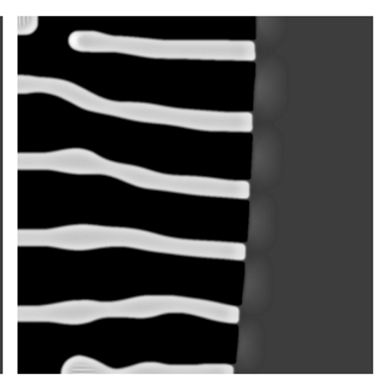

1.0

Рис. 7. Кинетика роста перлитной колонии при учете тангенциальных напряжений; $T=750 \mathrm{~K}, c_{0}=0.06,(d / L)^{2} K_{0}=-1.9 \mathrm{eV} / \mathrm{at}$.

С математической точки зрения, ветвление феррита (рис. 5) объясняется понижением эффективной поверхностной энергии феррита при сегрегации углерода вблизи межфазной границы. Подобный эффект был предсказан и экспериментально подтвержден [40-42] для случая, когда зеренная структура измельчается за счет сегрегации примеси на границах зерен. В работе [43] эффект Вейсмюллера был обобщен на межфазные границы, приводя к появлению равновесных дисперсных структур. В расчете (рис. 5) дилатации решетки обеспечивают энергетическую предпочтительность для удержания углерода вблизи феррита. Таким образом, наблюдаемая картина качественно подобна эффекту Вейсмюллера и предсказаниям модели [43].

При $\sigma_{n}=0$ распад развивается по классическому сценарию роста выделения феррита [27], и соответствующие расчеты здесь не приводим.

Для выявления роли тангенциальных напряжений $\sigma_{\tau}$ было проведено моделирование роста колонии при старте из однородного состояния с цепочкой эквидистантно расположенных выделений феррита и цементита на границе зерна (положение которой совпадает с границей расчетной области) при $\sigma_{n}=0$. Автокаталитическое появление такой цепочки выделений на ГЗ возможно при ускорении диффузии углерода на ГЗ по сравнению с объемом аустенита [15].

Из рис. 6 видно, что при $T=900 \mathrm{~K}$ даже небольшие $\sigma_{\tau}$ поддерживают устойчивый рост колонии, так что ламели ориентируются по нормали к фронту. В то же время упрощенная постановка задачи с раздельным описанием нормальных и тангенциальных напряжений не позволяет рассматривать зарождение новых ламелей в этом случае. На рис. 7 представлен рост колонии при наличии $\sigma_{\tau}$ и более низкой температуре $\left(T<T_{p}\right)$, когда автокаталитическое зарождение новых пластин происходит по механизму [18]. В этом случае при переходе к режиму стационарного роста выявляется оптимальное межпластиночное расстояние; на переходном этапе может иметь место осцилляция ширины пластин.

\section{4. Заключение}

Взаимодействие углерода в аустените с дилатациями решетки, создаваемыми растущей колонией перлита, стабилизирует рост колонии, приводя к более регулярным морфологиям распада. При этом нормальные напряжения на торцах колонии повышают температуру старта перлитного превращения, а тангенциальные напряжения обеспечивают устойчивость фронта колонии. Таким образом, ускорение диффузии на фронте колонии не является обязательным условием перлитного превращения. Замеченный механизм ветвления феррита за счет тенденции сегрегации углерода вблизи межфазной границы аустенит/феррит может иметь отношение как к перлитному, так и к бейнитному превращению.

Автор признателен профессору Ю.Н. Горностыреву, предложившему тему для исследования. 


\section{Приложение. Параметризация модели}

Энергии фаз рассчитывали с использованием экспериментальных данных для энергий растворения углерода [30], которые в отличие от приведенных в работах $[18,27]$ учитывают температурную зависимость: $\tilde{\varepsilon}_{F M(P M)}^{b c c}=\varepsilon_{F M(P M)}^{b c c}-\lambda_{\alpha} \tau, \tilde{\varepsilon}_{F M(P M)}^{f c c}=\varepsilon_{F M(P M)}^{f c c}-\lambda_{\gamma} \tau, \tau=T / T_{c}$, $\varepsilon_{F M}^{b c c}=1.1, \quad \varepsilon_{P M}^{b c c}=1.1, \quad \varepsilon_{F M}^{f c c}=-0.22, \quad \varepsilon_{P M}^{f c c}=0.38$, $\lambda_{\alpha}=0.46, \lambda_{\gamma}=0.14$ (eV/at), $T_{c}=1043 \mathrm{~K}$. Эти параметры согласуются с прежними вблизи $T \sim 1000 \mathrm{~K}$, т.е. в диапазоне перлитных температур.

Энергии смешения углерода принимали не зависящими от магнитного состояния, $v_{\alpha}=6, v_{\gamma}=1$ (eV/at) [44]. Отклонение свободной энергии цементита от свободной энергии $\alpha$-Fe было определено формулой $\Delta f_{\alpha \theta}=b_{0}+b_{1} \tau+b_{2} \tau^{2}, \quad$ где $b_{0}=0.09, \quad b_{1}=-0.13$, $b_{2}=0.04(\mathrm{eV} / \mathrm{at})$ [30,32]. Линия $T_{1}$ фазовой диаграммы, описывающая условие потери устойчивости $\gamma \rightarrow \theta$ при неизменной концентрации углерода, смещена влево (ср. с [18]) для качественного учета влияния напряжений на зарождение цементита вблизи феррита. При этом учитывался тот факт, что при $c \approx 0.10$ аустенит экспериментально не наблюдается [45]. Коэффициенты диффузии углерода в фазах были выбраны $D_{\alpha} / D_{\gamma}=D_{\gamma} / D_{\theta}=10$ в качественном согласии с данными $[46,47]$. Параметр $k_{c}$, определяющий ширину межфазной границы, определяли из условия $k_{c}^{2} /\left(k T L^{2}\right) \approx 7 \cdot 10^{-4}$ [48], откуда можно оценить размер образца $L \approx 1 \mu \mathrm{m}$.

Оценим также максимально возможное значение коэффициента $K_{0}$, определяющего величину напряжений. Величина упругой энергии из формул (2), (3) грубо оценивается как $f_{e l . i n} \sim(\sqrt{2} / 4) K_{0} \kappa^{2} c_{0}\left(c_{c o l}-c_{0}\right)(d / L)^{2}$, где $c_{c o l}$ - концентрация углерода в колонии (в феррите или цементите). С другой стороны, энергия, связанная с решеточными дилатациями в обозначениях [27] равна $f_{v}=A_{v} e_{v}^{2} / 2$, где $A_{v}=20 \mathrm{eV} / \mathrm{at}$, и для дилатации примем $e_{v}=\kappa\left(c_{c o l}-c_{0}\right)$. Отсюда следует:

$$
K_{0} \approx A_{v} \frac{\sqrt{2}}{2} \cdot \frac{\left(c_{c o l}-c_{0}\right)}{c_{0}} \cdot\left(\frac{L}{d}\right)^{2} .
$$

Используемые в расчетах значения $K_{0}$ предполагают частичную релаксацию напряжений и поэтому выбираются существенно ниже этой оценки.

\section{Список литературы}

[1] В.М. Счастливцев, Д.А. Мирзаев, И.Л. Яковлева. Перлит в углеродистых сталях. УрО РАН, Екатеринбург (2006). $311 \mathrm{c}$.

[2] R. Abbaschian, R. Reed-Hill. Physical Metallurgy Principles. SI Version. Cengage Learning (2009). 750 p.

[3] M.D. Graef, M.V. Kral, M. Hillert. J. Metals 58, 25 (2006).

[4] Fu-Wen Ling, D.E. Laughlin. Met. Trans. 10A, 921 (1979).
[5] A.T. Adorno, A.V. Benedetti, R.A.G. da Silva, M. Blanco. Ecletica Quimica 28, 33 (2003).

[6] A. Das, W. Gust, E.J. Mittemeijer. Mat. Sci. Tech. 16, 593 (2000).

[7] H. Ramanarayan, T. Abinandanan. Acta Mater. 52, 921 (2004).

[8] А.В. Пономарева, Ю.Н. Горностырев, И.А. Абрикосов. ЖЭТФ 147, 827 (2015).

[9] C. Zener. Met. Technol. 1, 1 (1946).

[10] M. Hillert. Jemkontorets Ann. 141, 757 (1957).

[11] D. Turnbull. Acta Met. 3, 55 (1955).

[12] B.E. Sundquist. Acta Met. 16, 1413 (1968).

[13] A. Yamanaka, T. Yamamoto, T. Takaki, Y. Tomita. MultiPhase-Field Study for Pearlite Transformation with Grain Boundary Diffusion. IV Int. Conf. Multiscale Materials Modeling (MMM2008) (2008), Florida, USA.

[14] В.Г. Вакс, А.Ю. Строев. ЖЭТФ 134, 113 (2008).

[15] В.Г. Вакс, А.Ю. Строев, В.Н. Урцев, А.В. Шмаков. ЖЭТФ 139, 1098 (2011).

[16] I. Steinbach, M. Apel. Acta Mater. 55, 4817 (2007).

[17] A. Hultgren. Trans. ASM 39, 915 (1947).

[18] I.K. Razumov, Yu.N. Gornostyrev, M.I. Katsnelson. Phys. Rev. Appl. 7, 014002 (2017).

[19] И.К. Разумов. ЖФХ 83, 1865 (2009).

[20] И.К. Разумов, Ю.Н. Горностырев, М.И. Кацнельсон. ФММ 118, 1 (2017).

[21] X. Zhang, T. Hickel, J. Rogal, S.Fähler, R. Drautz, J. Neugebauer. Acta Mater. 99, 281 (2015).

[22] K. Ankit, R. Mukherjee, T. Mittnacht, B. Nestler. Acta Mater. 81, 204 (2014).

[23] C.S. Smith. Trans. Am. Soc. Metals 45, 533 (1953).

[24] M. Hillert. The formation of pearlite / In: Decomposition of Austenite by Diffusional Processes/ Ed. V.F. Zackay, H.I. Aaronson. Interscience, N. Y. (1962). P. 197.

[25] S.A. Hackney, G.J. Shiflet. Acta Mater. 35, 1007 (1987).

[26] Б.Я. Любов. Кинетическая теория фазовых превращений. Металлургия, М. (1969). 264 с.

[27] I.K. Razumov, D.V. Boukhvalov, M.V. Petrik, V.N. Urtsev, A.V. Shmakov, M.I. Katsnelson, Yu.N. Gornostyrev. Phys. Rev. B 90, 094101 (2014).

[28] Дж. Смарт. Эффективное поле в теории магнетизма. Мир, M. (1968). 271 c.

[29] I.K. Razumov, Yu.N. Gornostyrev, M.I. Katsnelson. J. Phys.: Condens. Matter 25, 135401 (2013).

[30] Б.М. Могутнов, И.А. Томилин, Л.А. Шварцман. Термодинамика железо-углеродистых сплавов. Металлургия, М. (1972). $328 \mathrm{c}$.

[31] J.S. Darken, R.W. Gurry. J. Met. 3, 1015 (1951).

[32] A. Dick, F. Körmann, T. Hickel, J. Neugebauer. Phys. Rev. B 84, 125101 (2011).

[33] T. Mura. Micromechanics of Defects in Solids. Martinus Nijhoff Publishers, Dordrecht-Boston-Lancaster, (1987).

[34] C.B. Muratov. Phys. Rev. E 66, 066108 (2002).

[35] J.W. Cahn, J.E. Hilliard. J. Chem. Phys. 28, 258 (1958).

[36] K.G.F. Janssens, D. Raabe, E. Kozeschnik, M.A. Miodovnik, B. Nestler. Computational Materials Engineering. Elsevier, Amsterdam (2007), 360 p. 
[37] K.N. Tu, D. Turnbull. Acta Metall. 17, 1263 (1969).

[38] R.F. Mehl, W.C. Hagel. Prog. Metal. Phys. 6, 74 (1956).

[39] H.K.D.H. Bhadeshia. Bainite in steels. IOM Communications Ltd, London (2001). 460 p.

[40] J. Weissmuller. Nanostruct. Mater. 3, 261 (1993).

[41] E. Botcharova, J. Freudenberg, L. Schulz. Acta Mater. 54, 3333 (2006).

[42] J.R. Trelewicz, C.A. Schuh. Phys. Rev. B 79, 094112 (2009).

[43] И.К. Разумов. ФТТ 56, 749 (2014).

[44] H.K.D.H. Bhadeshia. J. Mat. Sci. 39, 3949 (2004).

[45] В.Г. Вакс, К.Ю. Хромов. ЖЭТФ 133, 313 (2008).

[46] Diffusion in Solids Metals and Alloys. Landolt-Börnstein New Series. V. III/26. Springer-Verlag, Berlin, 1990.

[47] B. Ozturk. Solid State Ionics 12, 145 (1984).

[48] S. Kartha, J.A. Krumhansl, J.P. Sethna, L.K. Wickham. Phys. Rev. B 52, 803 (1995). 